Commentary on The Case of "Hiro": Treating Tourette Syndrome by Comprehensive Behavioral Intervention for Tics (CBIT)

\title{
From Cook to Culinarian: Going Beyond the Manual When Delivering Behavior Therapy to Treat Tourette Disorder
}

\author{
BRIANNA WELLEN ${ }^{a}$ \& MICHAEL B. HIMLE ${ }^{a, b}$
}

\author{
${ }^{a}$ Department of Psychology, University of Utah, Salt Lake City, UT \\ b Correspondence regarding this article should be sent to Michael B. Himle, Department of Psychology, 380 S. \\ 1530 E. BehS \#502, University of Utah, Salt Lake City, UT 84112 \\ Email: michael.himle@utah.edu
}

\begin{abstract}
In the case of Hiro, Dr. Jeremy Lichtman (2017, this issue) describes how he flexibly employed a manualized behavior therapy protocol to successfully treat a child's involuntary motor and vocal tics. In doing so, he raises interesting observations and questions regarding manualized psychotherapy treatment protocols, including their intended role, the need for "flexibility with fidelity," and the strengths and limitations of following manuals in a step-by-step fashion. In our commentary, we draw upon Dr. Lichtman's experience in treating Hiro to highlight what we see as the two most important factors in Hiro's treatment: (1) the therapist's firm grasp of the underlying theory behind behavior therapy for tics, which allowed for flexibility in treatment delivery without jeopardizing treatment fidelity, and (2) the importance of having a strong foundational clinical skill set prior to beginning treatment.
\end{abstract}

Key words: Tourette Syndrome; Tics Disorders; Comprehensive Behavioral Intervention for Tics (CBIT); Habit Reversal Training (HRT); cognitive-behavioral training (CBT); protocol-based manualized therapy; principles-based manualized therapy; psychotherapy relationship skills; case studies; clinical case studies

Chronic tic disorders (CTD, including Tourette Disorder, TD) have long represented an orphaned class of puzzling, intractable, neurological conditions with unknown etiology. Although the exact cause of CTD remains unclear, there is considerable evidence that tics result from structural and functional abnormalities within the basal ganglia and associated structures (Albin \& Mink, 2006). Given their neurobiological etiology, treatment research has historically focused on developing and testing the efficacy and tolerability of various pharmacological ticsuppressing agents. While several medications have been found to be efficacious for reducing tics, none have been shown to result in complete symptom reduction. Furthermore, the use of the most efficacious drugs (e.g., typical and atypical antipsychotics) is often limited by concerns over side effects and long term use, especially in children (Whittington et al., 2016). More recently, a substantial evidence base has emerged that supports the efficacy of behavior therapy as a first-line treatment for reducing tics in both children and adults, offering patients and 
families an alternative or adjunct to pharmacotherapy (Himle, Woods, Piacentini, \& Walkup, 2006).

While behavioral treatments for CTD are not altogether new-habit reversal training, HRT, was developed and applied to the treatment of tics by behavioral psychologists Nathan Azrin \& Greg Nunn in the 1970s, see Azrin \& Nunn, 1973 - they have recently garnered more widespread recognition, acceptance, and use by psychologists and other clinical care providers. Recent advancements in dissemination have been aided by the publication of two large randomized controlled trials showing behavior therapy to outperform supportive therapy for reducing tics (Piacentini et al., 2010; Wilhelm et al., 2012). The publication of a step-by-step Comprehensive Behavioral Intervention for Tics (CBIT) treatment manual (Woods et al., 2008), which is the protocol that was used by Dr. Lichtman to treat the case of Hiro, has also helped advance dissemination. In his report, Dr. Lichtman provides a self-reflective account of how he flexibly employed CBIT to successfully treat Hiro’s tics. Below we comment on several issues that were raised in the case of Hiro by drawing on our own extensive experience using CBIT to treat CTD and training novice therapists in the CBIT protocol. We begin by commenting on the role of the CBIT manual in treating CTD, and the use of manuals in psychotherapy more generally. We then draw upon Dr. Lichtman's experience in treating Hiro to highlight what we see as the two most important factors in treatment success: (1) the therapist's firm grasp of the underlying theory behind CBIT, which allows for flexibility in treatment delivery without jeopardizing treatment fidelity, and (2) the importance of having a strong foundational clinical skill set prior to beginning treatment, which, in this case, undoubtedly saved the day.

\section{THE ROLE OF THE CBIT MANUAL IN TREATING CTD: THE RECIPE BOOK}

The publication of treatment manuals for a wide range of psychological disorders has become one of the defining features of the empirically-based practice movement (Task Force on Promotion and Dissemination of Psychological Procedures, 1995). While the very idea of manualizing psychotherapy has been scrutinized (see Addis, Wade, \& Hatgis, 1999), and the potential pitfalls of inflexible adherence to manualized protocols has been extensively discussed in the literature (Addis, 1997), when used appropriately, manuals can be an invaluable tool for guiding both novice and experienced therapists as they administer (or learn to administer) empirically-based treatments (Addis, 1997).

In some ways, treatment manuals can be viewed as recipe books that outline the essential or recommended therapeutic techniques (i.e., the ingredients), materials such as therapy forms (i.e., the pots and pans), and methods (e.g., ordering and mixing of ingredients, recommended cooking time and temperature, etc.). If followed with enough fidelity, employing the treatment by carefully following the manual will likely result in an approximation of the proven outcome, such as the average degree of symptom reduction observed in a clinical trial (or, a delicious and perfectly risen cake in our cooking metaphor).

However, what if the recipe doesn't quite fit the project? This is often the case when using a treatment manual when delivering psychotherapy for clinical problems that are 
admittedly more complicated than even the most sophisticated cooking recipe. Take, for example, the second author's (MBH's) recent failed attempt to bake his daughter a large birthday cake resembling the Death Star from Star Wars, which is in the shape of a large sphere. Unfortunately, hours of searching failed to turn up a Death Star cake recipe, so he decided to use generic cake recipe and do his best; after all, he had baked several small square cakes before with some degree of success. Not surprisingly, naively modifying the recipe (tripling some ingredients while leaving others out, increasing the cooking temperature) resulted in an inedible mess, not to mention a distraught 9-year-old girl. What went wrong? One obvious answer is that the existing recipe was flawed. However, beyond blaming the recipe, the most important factor is that there was a mismatch between the complexity of the project and the skill and knowledge of the cook. In this example, the cook was attempting to flexibly apply the recipe to bake a cake for which the recipe was not sufficiently delineated and without the theoretical knowledge to inform him on how to adjust the recipe as needed.

For the beginning cook who dabbles in the kitchen at home, recipes serve as a sufficient guide for preparing most basic meals. In such cases, basic cooking knowledge and skill are sufficient if no modifications are required. If one follows the steps in the recipe, incorporates the primary ingredients, and cooks the meal at the right temperature for the suggested length of time, the meal will most likely approximate the desired outcome and be acceptable to the consumer. However, if one deviates too far from the recipe (e.g. in order accommodate the needs of their 9year-old Star Wars fanatic) by leaving out essential ingredients or accelerating/decelerating the cooking time or temperature, disappointment ensues.

This begs the question: what if one wants to tackle a more sophisticated project for which existing recipes are insufficient? This is where culinary training and experience are important. As opposed to the amateur cook, culinarians are extensively trained in the art and science of preparing, cooking, and presenting food. Through this training, they develop a sophisticated knowledge of the chemistry and biology of food, including how ingredients interact, break down, are affected by varying lengths and degrees of heat, etc. With this expert knowledge of the mechanisms and processes involved in combining and cooking food ingredients, which comes from extensive training and applied experience, culinarians are thus better able to conceptualize how to approach more complex cooking projects, anticipate the positive and negative outcomes likely to result from modifying a recipe, and generate entirely new recipes altogether.

So what does all of this have to do with Dr. Lichtman and Hiro? First, Hiro’s case demonstrates the importance of having a thorough working understanding of behavioral theory prior to implementing CBIT (i.e., becoming a behavioral/CTD/CBIT culinarian). Second, Hiro's case highlights the importance of viewing CBIT as a collection of specific therapeutic techniques that are delivered via a broader therapeutic skill set. This involves developing a working case formulation, attending carefully to the therapeutic relationship, and ensuring that the patient, family, and therapist are on the same page regarding expectations and goals for therapy. In other words, implementing CBIT, or any treatment manual for that matter, requires a strong foundation of requisite clinical skills that are not outlined in the manual. We discuss each of these points as they related to Hiro's case in more detail below. 


\section{THE ROLE OF THEORY: BECOMING A CULINARIAN}

Dr. Lichtman has provided a concise review of the behavioral model of TS, and we encourage readers interested in treating CTD to digest that section, and the accompanying references carefully. Those who understand the theory behind a behavioral model of tics will quickly come to understand that CBIT is a collection of theory-driven, empirically supported behavior analytic techniques that are designed to identify and alter the function of tic behaviors, thereby decreasing their frequency, intensity, and interference. In addition, consistent with the behavior analytic approach upon which CBIT is based, it is assumed that the degree to which various internal (e.g., premonitory urges, mood states) and external (e.g., settings, activities, social reactions) antecedents and consequences serve to attenuate or exacerbate tics will be highly individualized based on the individual's learning history. Given the heterogeneity of tics, not to mention the complexity added when comorbidity is present, an individualized and flexible approach to treatment is almost always necessary and requires the clinician to be a CTD and CBIT culinarian capable of adapting treatment delivery based on his/her client's presentation and needs.

Hiro's case is a nice example of the limitations of treatment manuals for teaching novice therapists how to adapt treatment for any particular individual while maintaining fidelity to treatment. It also demonstrates the risks associated with inflexible adherence to the manual. In the first several sessions, Dr. Lichtman describes (see sessions 3-5) that he was "strictly adhering to the manual, adding new elements, such as relaxation techniques, as stipulated” (2017, p. 18) and that he was doing so "despite any evidence that it was effective for Hiro" (see session 5; 2017, p. 18). As a result, Hiro’s symptoms were not improving, his compliance was poor, and his parents were losing faith in treatment. Fortunately, Dr. Lichtman recognized that "something had to change for treatment to succeed” (see session 5; 2017, p. 18).

Rather than abandoning CBIT altogether, the treatment team re-formulated the case and began using the CBIT treatment protocol flexibly while maintaining fidelity to the behaviorally based approach to treating CTD (see Hamilton, Kendall, Gosch, Furr, \& Sood, 2008 for more on the general topic of "flexibility within fidelity" in manualized treatment delivery). Specifically, the treatment team (1) de-emphasized the step-by-step function-based assessment elements of treatment in favor of focusing more time and effort on habit reversal training (HRT), (2) increased their focus on how Hiro's ADHD was affecting his ability to learn and use HRT, (3) began focusing on more general (i.e., nonspecific) elements of treatment such as incorporating Hiro's interests into treatment and focusing on the therapeutic relationship, and (4) increased the parents' involvement in treatment. As Dr. Lichtman notes, advice for how to make these necessary adjustments are not explicitly outlined in the CBIT manual. Rather, they required a careful and ongoing case formulation and a thorough understanding of behavioral theory and the behavioral model of tics, which helped the treatment team to decide both when and how to "flex" treatment without sacrificing treatment fidelity. To better understand how theory helped problem solve Hiro’s case, we will consider several of the treatment challenges Dr. Litchman faced, how they were solved, and offer some additional "food for thought." 
Dr. Lichtman notes in his discussion that, “For Hiro’s case, there were treatment elements - like the Function-Based Assessment - that were unnecessary and proved unwieldy" (2017, p. 26). To phrase this differently, implementing the function-based assessment in lockstep fashion did not reveal any consistent or significant tic-exacerbating external antecedents or consequences that could be meaningfully (or practically) targeted for intervention. Once he realized this, Dr. Lichtman made an informed decision to deemphasize this particular therapeutic technique and move on to teaching Hiro to manage his tics using habit reversal training (HRT).

But the function-based assessment is a primary ingredient in CBIT, so why didn't it work? Consistent with taking an individualized behavior-analytic approach to treatment, perhaps it is the case that Hiro's learning history was such that external antecedents and consequences were not exerting a strong influence on his tics. For example, there may have been no consistent history of people reacting to his tics, Hiro escaping tasks for ticcing, etc. We have certainly experienced this in some of the children we have treated over the years. If this was indeed the case that Hiro's learning history was not powerfully impacting his tics, moving on from the function-based elements of treatment early is a justifiable treatment decision, especially given that the parents seemed to be losing patience with the pace and progress of treatment (which we address in more detail below). While not explicitly stated, it seems apparent to us that, in Hiro's case, the decision to move on from the function-based component of CBIT was well informed by the treatment team's case formulation, data that was gathered during the first few sessions, and the observation that other elements of treatment (e.g., HRT) were demonstrating some effectiveness. Extending our metaphor, a culinarian, or an experienced therapist, would rely on their expert knowledge and experience to decide whether an in depth function-based assessment was a necessary ingredient for treatment success.

While the function-based component of treatment might not have been necessary in this case, that does not imply that it is useless or a waste of time. It is important to be mindful of the rationale and purpose of the function-based component of CBIT. The rationale for the functionbased approach is that although tics are not caused by environmental variables, tic-contingent antecedents and consequences can serve to exacerbate tics and lead to unnecessary interference and functional impairment. The primary goal of the function-based assessment is to identify factors associated with problematic tic exacerbations. This is not sufficiently described in the manual. The second author (MBH) has trained many novice CBIT therapists over the past 10 years and frequently encounters therapists who spend considerable time in treatment attempting to address each and every tic-exacerbating factor. This is often unnecessary and counterproductive, as it was in Hiro's case. For example, if a child's tics are transiently worse when he/she is playing videogames, but this is not causing any problems in his overall daily functioning, it not likely worth taking away the child's videogames, especially if you want him to stay in treatment. On the other hand, if videogames are exacerbating tics to the point that he cannot complete schoolwork later that evening, then this should be addressed by, for example, scheduling videogame time for after homework is complete. By focusing only on problematic tic exacerbations rather than on all tic exacerbations, the therapist can concentrate his/her efforts in a way that is more productive. 
Even when problematic tic exacerbations are not identified during the function-based assessment, there are several reasons that it can still be useful for clinicians to take some time to teach the family about the function-based model. First, like Hiro, most children who successfully complete CBIT continue to have occasional tics, and new tics often emerge after treatment has ended. We often find that it is useful for parents and children to understand that although tics are involuntary, they can be influenced by context, social reactions, etc. In other words, it is often useful, for therapists to help patients and parents to understand the idea or the theory behind the function-based approach. This will increase mindfulness of how their reactions might influence tics in the future and encourage them to take steps to create a "tic neutral" environment in which tics do not receive special attention or accommodation, which could function to make the tics worse.

Second, it is not uncommon for families who appreciate the behavioral model to later in therapy spontaneously identify function-based variables that are exacerbating tics that were missed in earlier sessions.

Third, it seems that the function-based assessment did provide potentially useful information in this case. In particular, Hiro's family noted that his tics tended to be worse after school. It is quite possible that the tics were being exacerbated by a particular mood state (excitement upon arriving home, boredom, stress from the school day), parental or sibling attention, or cognitive fatigue. It is not clear whether such possibilities were explored in Hiro's case. Regardless, there is no reason to expect, from a purely biological model based on our current understanding of the CTD brain, that tics would occur more frequently in one setting than another.

Fourth, while in Hiro’s case there might not have been any particular tic-exacerbating consequences identified during the function-based assessment, knowing that his tics tended to increase after school could also provide the therapist with important information such as when to practice HRT (e.g., 15 minutes of practice after school when tics are at their worst).

Finally, it's worth noting that while the function-based elements of CBIT might not have been particularly relevant for Hiro, results from the child CBIT randomized controlled trial found that out of 51 children randomized to CBIT, all participants endorsed at least two ticexacerbating antecedents and all but two endorsed at least one tic-exacerbating consequence (Himle et al., 2014). These findings strongly suggest that not taking the time to conduct a thorough functional assessment could, more times than not, result in the therapist missing out on important treatment-related information.

In reading about Hiro's treatment, an additional thought that came to mind regarding the function-based intervention component of CBIT concerns the role of relaxation. It was noted that relaxation was not a particularly useful tool for Hiro in terms of reducing his tics. The rationale for including relaxation in the CBIT protocol was that most individuals with CTD report that their tics are exacerbated by stress and anxiety (Silva, Munoz, Barickman, \& Friedhoff, 1995) and research shows that while relaxation is not a particularly efficacious standalone treatment, it has been shown to reduce tics, at least temporarily, for some children (Bergin, 
Waranch, Brown, Carson, \& Singer, 1998; Peterson \& Azrin, 1992), thus making it a potentially useful tic management tool. In our clinical experience, we find that many children with CTD find it useful to use relaxation techniques during periods of heightened stress or anxiety (e.g., before taking a test) because, in addition to making them feel better, it temporarily reduces their tics so that they are less noticeable and/or interfering with the task at hand (e.g., completing the test). In this way, relaxation can be conceptualized as a function-based strategy to reduce the impact on tics on daily life, which is consistent with the idea that CBIT teaches a set of tic management skills.

The importance of understanding of behavioral theory and the behavioral model of tics was also exemplified in several other elements of Hiro's treatment. First, a decision was made early in the course of treatment not to include a behavioral reward program for treatment engagement and homework completion, as outlined in the CBIT protocol, in favor of attempting to "develop in Hiro a sense of internal motivation" (2017, p. 16). While it is true that extrinsic rewards can undermine intrinsic motivation (Deci, Koestner, \& Ryan, 1999), we are reminded that Hiro reported that he was not very bothered by his tics and he certainly was not seeking treatment on his own accord. As is common in CTD, Hiro's parents were much more worried about his tics and future than he was. While developing intrinsic motivation is an admirable goal, from Hiro's perspective, he was being asked to engage in difficult tasks, such as counting tics and engaging in a competing response that likely made him uncomfortable because he had to tolerate aversive premonitory urges, and required him to stop engaging in preferred activities. He had to stop playing videogames to engage in a competing response, and come to sessions rather than play outside. On the other hand, ticcing takes relatively little effort, works well to relieve unpleasant urges, and is not currently causing much of a problem, emphasizing the importance of reinforcement programs for some children. Not surprisingly, treatment engagement increased when the reward program was later introduced, suggesting that the reward program, which is recommended in the CBIT manual, may have been an important part of the recipe in order to maintain fidelity.

Finally, Dr. Lichtman accurately notes that the CBIT manual contains relatively few examples of competing responses for tics, which required him to use his creativity to come up with his own. While this criticism of the manual is fair, it would be nearly impossible to generate competing responses for each and every possible movement that a child can make. Rather, the manual assumes that teaching the clinician the behavioral principles behind HRT and the "rules" for competing response training will be sufficient to help the clinician come up with effective competing responses for a wide range of tics. As long as the competing response follows the outlined rules, learning can take place and the competing response should be effective. This is an area where Dr. Lichtman is commended, and is yet another good example of how a firm grasp of the underlying theory can help the therapist problem-solve issues that are not explicitly outlined in the treatment manual. 


\section{THE IMPORTANCE OF A STRONG AND VARIED THERAPEUTIC SKILL SET}

The second important lesson learned from the case of Hiro is the importance of viewing CBIT (or any behavioral treatment) as a collection of specific therapeutic techniques, the delivery of which requires a strong and broad foundation of clinical skills and knowledge that are assumed to be in place when the therapist pulls the manual off the shelf to begin treatment. These non-CBIT-specific elements are skills that go well beyond what is taught in the CBIT treatment manual and highlight the importance of rigorous training and supervision in foundational clinical competencies. Regarding this topic, several important lessons can be gleaned from the case of Hiro.

Hiro's case demonstrates that treating CTD is rarely straightforward. Perhaps the most daunting challenge is figuring out how to manage comorbidity. As Dr. Lichtman notes, CTD is known to have high rates of comorbidity with attention-deficit hyperactivity disorder (ADHD), obsessive compulsive disorder (OCD), anxiety and mood disorders, oppositional defiant disorder, and a host of other problems including impulsivity, anger problems, specific learning problems, and sleep disturbance, among others (Freeman, Fast, Burd, Kerbeshian, Robertson, \& Sandor, 2007).

When comorbidity is present, it can pose several challenges to the CTD therapist. First, differential diagnosis can be challenging. As we saw with Hiro, for example, complex tics are often difficult to distinguish from OCD-related compulsions, yet this is an important differential diagnosis to make because it has implications for the treatment protocol one chooses (e.g., CBIT for tics or exposure and response prevention for OCD). Second, comorbidity can make it difficult to prioritize treatment targets and goals. Several studies have shown that internalizing and externalizing symptoms are uniquely associated with impairment across most psychosocial domains, including decreased self-esteem, family burden, relationship problems, and decreased quality of life (Conelea et al., 2011). As such, it is generally recommended that such symptoms be the initial focus of treatment because they are not addressed by CBIT. However, it is not uncommon for children and parents to disagree about which symptoms - both in terms of comorbidity or which tics - are causing the biggest problem, making it difficult to agree to a specific treatment plan. Finally, when tics are the focus of treatment, comorbidity can complicate CBIT. For example, depression can decrease motivation to engage in treatment and noncompliance can lead to treatment resistance.

In Hiro's case, as is common in children with ADHD who are learning CBIT, problems with inattention and impulse control were making it difficult for him to focus during sessions and to catch his tics during HRT. This resulted in treatment taking a bit longer to work and giving Hiro's parents the impression that Hiro was not willing to practice HRT skills and/or that treatment was not working. Recognizing how Hiro’s ADHD was affecting treatment and making necessary adjustments to treatment turned out to be essential to Hiro’s success in several ways.

First, Dr. Lichtman recognized the need to adjust his approach to increase Hiro's engagement in treatment. He did so largely by tapping into Hiro's interests in a particular video 
game. This was a brilliant move as research has shown that children with ADHD respond best to frequent reward (Tripp \& Alsop, 1999). By tapping into his interests in videogames, treatment seemed to be much more rewarding to Hiro, thus helping to hold his attention and increase his engagement in an otherwise mundane task.

Second, Dr. Lichtman recognized that he was providing a high level of structure and direction in the therapy session, essentially functioning as Hiro's surrogate frontal lobe, which helped to explain why Hiro was better able to use HRT skills in session than at home. This led him to recognize the importance of increasing parental involvement in treatment so that Hiro's parents could more effectively assist him with HRT practice outside of sessions. In fact, this is one reason why parental involvement is explicitly encouraged (if not essential) in all cases of CBIT. Another reason to involve parents throughout the course of treatment is that CBIT is considered a family-based intervention designed to teach both children and their parents a set of skills for better managing current tics as well as those that might arise in the future. We frequently tell parents that our goal is to teach the parents how master CBIT so that we can make ourselves as useless as possible as quickly as possible. This approach helps to increase parental engagement in treatment, teaches them how to appropriately respond to their child's tics and reinforce CBIT skills, and ensures that they are practicing HRT correctly. Although the parents were eventually incorporated into HRT instruction in later sessions (in anticipation of Hiro moving away), it is not clear whether parents were involved in early sessions, for example to provide social support (one of one of the three primary elements of HRT) by prompting and praising the use of competing responses. If the parents were not involved in early sessions, this was a curious protocol deviation that was not justified or explained in the case report. We can't help but wonder whether lack of parental involvement early in treatment might have contributed to some of the challenges faced in the first few sessions, especially as they relate to parental buyin and expectations.

In our experience, "buy-in” issues are most common in parents who do not fully understand their role in treatment, who do not understand the underlying rationale or the behavioral model upon which CBIT is based, and/or those who have unrealistic or uninformed expectations for treatment. Regarding the parental role in treatment, we occasionally run into parents who wish to drop their child off so that they can be "treated," like dropping a child off for piano lessons. This approach does not work for treating tics with the CBIT approach. When this happens, we often spend considerable time in the first few sessions explicitly outlining the parents' role in treatment and, at the end of each session, both the parent(s) and the child are provided with a "to do" list of activities for the week. Most parents quickly realize that they will be putting in just as much work as their child and that their effort will determine the pace and success of therapy. While some parents balk at this element of treatment, we have found that most do not, as long as they understand the rationale, based on the behavioral model, for their involvement and the expectation (or insistence) that they be involved is made early in the course of treatment. In our clinical experience, this is an ingredient in the CBIT protocol that we would definitely not leave out.

Regarding treatment expectations, it is important for parents to know that the goal of CBIT is not to cure or eliminate tics completely, but rather to teach tic management skills that, if 
practiced consistently, will likely reduce the frequency of tics and/or decrease the extent to which tics cause functional interference. In fact, research shows us that not all children respond to treatment and for those who do, most do not experience complete elimination of tics. For example, results from the aforementioned randomized controlled trial comparing CBIT to supportive therapy showed that 53\% of children receiving CBIT demonstrated a clinically significant improvement in symptoms (compared to 19\% in PST) with a mean 31\% reduction in tic severity after CBIT treatment (Piacentini et al., 2010). Stated differently, almost half of children did not respond to treatment (why this might have been the case is beyond the scope of this commentary) and most, if not all, of those who did respond continued to exhibit tics.

We can't help but wonder whether some of the early struggles that Dr. Lichtman faced were due to the parent's lack of involvement early in treatment and/or unrealistic expectations for treatment, and would welcome more comment on that issue. What is clear, however, is that Dr. Litchman's strong existing clinical skills set allowed him to go "beyond the manual" to make adjustments to therapy that played no small part in Hiro's success. These included (1) the ability to form a strong clinical bond with Hiro and his parents, especially by incorporating Hiro's interests to make treatment fun; (2) the ability to recognize signs of a rupture in the therapeutic alliance and to make necessary adjustments to treatment to re-engage Hiro and his parents; (3) the ability to conduct a thorough clinical assessment and differential diagnosis; and (4) the ability to make procedural adjustments to the manualized HRT protocol, based on Hiro's ADHD, to more effectively activate the underlying treatment mechanism. As Dr. Lichtman notes, these are not elements that are outlined in the CBIT manual, but rather rely on a foundation of clinical skills that were developed through years of graduate training and clinical supervision.

In summary, in the case of Hiro, Dr. Lichtman eloquently describes how he flexibly employed CBIT to successfully treat Hiro's tics. We would be remiss not to comment on how fortunate Hiro and his parents were to have such a dedicated, knowledgeable, and thoughtful treatment team. What is particularly impressive about Dr. Lichtman's case report is how he used case formulation, hypothesis testing, and collaborative decision-making to optimize outcomes when delivering manualized treatment for CTD. In doing so, he illustrates the challenges facing both novice and experienced therapists who have the fortitude to tackle this complex and highly varied disorder while simultaneously highlighting the strengths and limitations of the CBIT approach. More importantly, Dr. Lichtman's case study reminded us of several broader issues regarding the use of step-by-step treatment manuals when delivering interventions from the trenches, including the essential role of nonspecific factors in all forms of psychotherapy and the importance of incorporating developmental, family, and interpersonal (among other) factors into treatment planning. As Dr. Lichtman points out, these broader issues are not always adequately addressed in manualized treatments, yet can play an instrumental role in the success of treatment.

\section{REFERENCES}

Addis, M.E. (1997). Evaluating the treatment manual as a means of disseminating empirically validated psychotherapies. Clinical Psychology: Science and Practice, 4, 1-11. 
Addis, M.E., Wade, W.A., \& Hatgis, C. (1999). Barriers to dissemination of evidence-based practices: Addressing practitioners’ concerns about manual-based psychotherapies. Clinical Psychology: Science and Practice, 6, 430-441. doi:10.1093/clipsy.6.4.430.

Albin, R.L., \& Mink, J.W. (2006). Recent advancements in Tourette syndrome research. Trends in Neurosciences, 29, 175-182. doi:http://dx.doi.org/10.1016/j.tins.2006.01.001.

Azrin, N.H., \& Nunn, R.G. (1973). Habit-reversal: A method of eliminating nervous habits and tics. Behaviour Research \& Therapy, 11, 619-628.

Bergin, A., Waranch, H.R., Brown, J., Carson, K., \& Singer, H.S. (1998). Relaxation therapy in Tourette syndrome: A pilot study. Pediatric Neurology, 18, 136-142.

Conelea, C.A., Woods, D.W., Zinner, S.H., Budman, C., Murphy, T., Scahill, L., ... Walkup, J. (2011). Exploring the impact of chronic tic disorders on youth: Results from the Tourette syndrome impact survey. Child Psychiatry \& Human Development, 42, 219-242. doi:10.1007/s10578-010-0211-4.

Deci, E.L., Koestner, R., \& Ryan, R.M. (1999). A meta-analytic review of experiments examining the effects of extrinsic rewards on intrinsic motivation. Psychological Bulletin, 125, 627-688. doi:http://dx.doi.org/10.1037/0033-2909.125.6.627.

Freeman, R.D., Fast, D.K., Burd, L., Kerbeshian, J., Robertson, M.M., Sandor, P. (2007). An international perspective on Tourette syndrome: selected findings from 3500 individuals in 22 countries. Developmental Medicine and Child Neurology, 42, 436-447. doi: 10.1111/j.1469-8749.2000.tb00346.x

Hamilton, J.D., Kendall, P.C., Gosch, E., Furr, J.M., \& Sood, E. (2008). Flexibility within fidelity. Journal of the American Academy of Child \& Adolescent Psychiatry, 47, 987993.

Himle, M.B., Capriotti, M.R., Hayes, L.P., Ramanujam, K., Scahill, L., Sukhodolsky, D.G., ... Piacentini, J. (2014). Variables associated with tic exacerbation in children with chronic tic disorders. Behavior Modification, 38, 163-183. doi:10.1177/0145445514531016.

Himle, M.B., Woods, D.W., Piacentini, J.C., \& Walkup, J.T. (2006). A brief review of habit reversal training for Tourette syndrome. Journal of Child Neurology, 21, 719-725.

Lichtman, J.D. (2017). The case of "Hiro": Treating Tourette Syndrome by Comprehensive Behavioral Intervention for Tics (CBIT). Pragmatic Case Studies in Psychotherapy, 13(1), Article 1, 1-37. Available: http://pcsp.libraries.rutgers.edu

Peterson, A.L., \& Azrin, N.H. (1992). An evaluation of behavioral treatments for Tourette syndrome. Behaviour Research \& Therapy, 30, 167-174.

Piacentini, J. C., Woods, D. W., Scahill, L. D., Wilhelm, S., Peterson, A., Chang, S.,...Walkup, J. T. (2010). Behavior therapy for children with Tourette Syndrome: A randomized controlled trial. Journal of the American Medical Association, 303, 1929-1937. doi:10.1001/jama.2010.607.

Silva, R.R., Munoz, D.M., Barickman, J., \& Friedhoff, A.J. (1995). Environmental factors and related fluctuation of symptoms in children and adolescents with Tourette's disorder. Journal of Child Psychology and Psychiatry, 36, 305-312. doi: 10.1111/j.14697610.1995.tb01826.x

Task Force on Promotion and Dissemination of Psychological Procedures (1995). Training in and dissemination of empirically validated treatments. The Clinical Psychologist, 48, 323. 
Tripp, G., \& Alsop, B. (1999). Sensitivity to reward frequency in boys with attention deficit hyperactivity disorder. Journal of Clinical Child Psychology, 28, 366-375.

Whittington, C., Pennant, M., Kendall, T., Glazebrook, C., Trayner, P., Groom, M., ...Hollis, C. (2016). Practitioner review: Treatments for Tourette syndrome in children and young people- a systematic review. Journal of Child Psychology \& Psychiatry, 57, 988-1004. doi:10.1111/jcpp.12556.

Wilhelm, S., Peterson, A.L., Piacentini, J., Woods, D.W., Deckersbach, T., Sukhodolsky, D.G., . . . Scahill, L. (2012). Randomized trial of behavior therapy for adults with Tourette syndrome. Archives of General Psychiatry, 69, 795-803. doi: 10.1001/archgenpsychiatry.2011.1528.

Woods, D. W., Piacentini, J. C., Chang, S. W., Deckersbach, T., Ginsburg, G. S., Peterson, A. L., . . . Wilhelm, S.. (2008). Managing Tourette Syndrome: A behavioral intervention for children and adults: Therapist Guide. New York: Oxford University Press. 